\title{
Extending dark optical trapping geometries
}

\author{
Aidan S. Arnold \\ Department of Physics, SUPA, University of Strathclyde, Glasgow G4 ONG, UK (aidan.arnold@strath.ac.uk)
}

Received March 14, 2012; accepted April 21, 2012;

posted April 25, 2012 (Doc. ID 164732); published June 20, 2012

\begin{abstract}
New counterpropagating geometries are presented for localizing ultracold atoms in the dark regions created by the interference of Laguerre-Gaussian laser beams. In particular dark helices, an "optical revolver," axial lattices of rings, and axial lattices of ring lattices of rings are considered and a realistic scheme for achieving phase stability is explored. The dark nature of these traps will enable their use as versatile tools for low-decoherence atom interferometry with zero differential light shifts. (c) 2012 Optical Society of America

OCIS codes: $020.1335,020.7010$.
\end{abstract}

The optical dipole force generated by far off-resonance laser beams has been utilized in an extremely wide variety of experiments on ultracold matter. It has been used as a matter wave beam splitter/tunable barrier [1,2], has stirred up vortices [3], and created crystals of atoms stored in precise optical lattices [4]. If the light is reddetuned from an atomic resonance atoms are attracted to regions of bright optical intensity, whereas bluedetuned light attracts atoms to the lowest intensity regions.

Smoothly varying electric fields can have a zero crossing in space, and one can thereby make spatial regions in one, two, or three dimensions that are completely dark. The advantage of this darkness is that as the atoms scatter photons at a rate proportional to $I / \Delta^{2}$, where $I$ is the light intensity and $\Delta$ is the detuning from resonance, ultracold atoms in dark traps have a greatly reduced heating rate due to photon absorption and emission.

In addition, the light shift due to the optical dipole effect is proportional to $I / \Delta$, and thus two regions of different intensity $I_{1}$ and $I_{2}$ will have corresponding energy difference $E_{2}-E_{1}$, leading to an (often unwanted) timedependent phase shift $\phi=\left(E_{2}-E_{1}\right) t / \hbar$. Such a problem is obviated in dark traps. Gravitational forces and thermal motion/quantum mechanical zero-point energy will lead to sampling of nondark regions of the potential; however, magnetic "levitation" [2] and ultralow temperatures, respectively, can minimize these effects.

To illustrate the wide range of options available with optical trapping, we discuss some of the geometries for atom trapping obtained to date. Single-beam bluedetuned traps can be used as "optical pipes" [5] via Laguerre-Gauss (LG or "donut") laser modes [6]. By combining two copropagating laser beams, one can also make three-dimensional (3D) dark traps "optical bottles" $[\underline{7}, \underline{8}]$, rings [9] $[\underline{10}, 11]$. To date counterpropagating geometries with LG beams have been relatively rarely used experimentally, although they open a plethora of geometries as we show here. Cylindrical axicon beams without the angular momentum of LG beams were used in a counterpropagating geometry in Ref. [12] to create a dark axial array of rings ("axial" refers to the beam propagation direction). Theoretical papers have discussed ways to make bright (red-detuned) axial lattices of ring lattices [13], and helices [14], and we extend all of these geometries into the preferable (low-decoherence and light shift) dark configuration.
The LG field mode of index $\ell$ (only the case with the other index $p=0$ is used) can be expressed in cylindrical polars as

$$
\begin{aligned}
L G_{\ell}= & \sqrt{\frac{2^{1+|\ell|} \operatorname{Pr}^{2|\ell|}}{\pi w^{2(1+|\ell|)}|\ell| !}} \exp \left[i s k z\left(1+\frac{r^{2}}{2\left(z^{2}+z_{R}^{2}\right)}\right)\right] \\
& e^{-r^{2} / w^{2}-i \ell \theta-i \omega t-i s(|\ell|+1) \arctan \left(z / z_{R}\right)},
\end{aligned}
$$

where the beam has wavenumber $k=2 \pi / \lambda$, angular frequency $\omega=c k$, power $P$, waist $w_{0}$, Rayleigh range $z_{R}=\pi w_{0}^{2} / \lambda, w(z)=w_{0} \sqrt{1+z^{2} / z_{R}^{2}}$ and $s= \pm 1$ is the propagation direction relative to the $z$ axis. In the following illustrations we will consider rubidium atoms in light fields of wavelength $\lambda=532 \mathrm{~nm}$, far blue-detuned from the atomic resonances at $\lambda_{0}=780 \mathrm{~nm}$ and $795 \mathrm{~nm}$. All beam waists are $w_{0}=5 \mu \mathrm{m}$, unless stated otherwise. Note the mode described in Eq. (1) is the square-root of the intensity (i.e., not the electric field, but proportional to it). Useful estimates of the scattering rate and potential depth of a dipole beam light with intensity $I$ are [10]

$$
(R, U) \approx \hbar \Gamma \beta\left(\Delta_{\Gamma}^{-2},\left(k_{B} \Delta_{\Gamma}\right)^{-1}\right) / 8,
$$

respectively, where $\beta=I / I_{S}$ and the Rb saturation intensity and natural linewidth are $I_{S}=16.3 \mathrm{~W} / \mathrm{cm}^{2}$ and $\Gamma=2 \pi \times 6 \mathrm{MHz}$, respectively. The detuning of the dipole beam in linewidths is $\Delta_{\Gamma}=\left(\omega-\omega_{0}\right) / \Gamma \approx 3.0 \times 10^{7}$.

Examples of the different kinds of dark optical trap geometries obtainable using superpositions of LG beams are: dark helix lattices (Fig. 1), counter-rotating dark helix lattices (Fig. 2), an axial lattice of rings (Fig. 3), an axial lattice of ring lattices (Fig. 4), and an axial lattice of ring lattices of rings (Fig. 5).

Tonks-Girardeau gases are intriguing systems where 1D confinement of strongly interacting bosons can lead to behavior mimicking noninteracting fermions [15]. In Fig. 1 (similar to Fig. 2), each helical dark core has a lead angle $\approx \frac{\lambda\left(\ell_{1}-\ell_{2}\right)}{\sqrt{2} \pi w_{0}\left(\sqrt{\ell_{1}}+\sqrt{\ell_{2}}\right)}=39 \mathrm{mrad}$ and has harmonic trap frequencies $154 \mathrm{kHz}$ and $6.0 \mathrm{kHz}$ in the axial and radial directions, respectively. By reducing the detuning (to tighten the trap) it will be possible to use each helix to compactly store long Tonks gas chains-in Fig. 1 even a $40 \mu \mathrm{m}$ axial extent of helix with radius $11 \mu \mathrm{m}$ has an uncoiled length of $1 \mathrm{~mm}$. Such an extension is not 

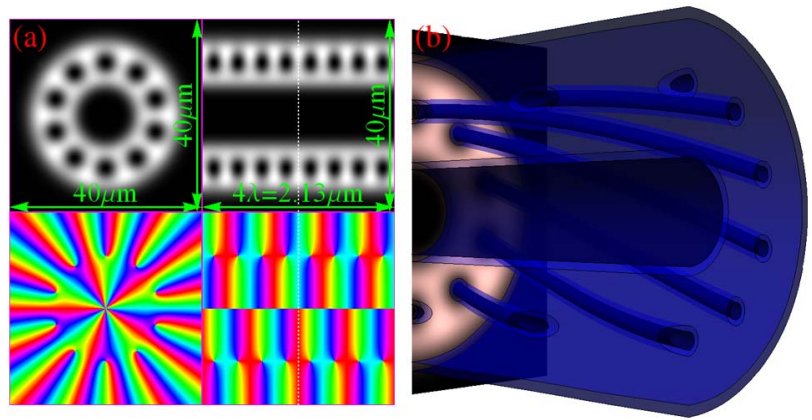

Fig. 1. (Color online) A dark helix lattice arising from a superposition of $100 \mathrm{~mW}$ of $\mathrm{LG}_{5}$ and a counterpropagating $170 \mathrm{~mW}$ $\mathrm{LG}_{15}$ beam. (a) Contains radial [left $(40 \mu \mathrm{m})^{2}$ ] and axial [right $(4 \lambda=2.13 \mu \mathrm{m}) \times 40 \mu \mathrm{m}]$ slices through the beam. Intensity and phase are shown in the top and bottom images, respectively. At the maximum intensity shown (white zones) the scattering rate and potential depth are $R=0.19 \mathrm{~Hz}$ and $U=43 \mu \mathrm{K}$, respectively. (b) Extends the radial $2 \mathrm{D}$ intensity plot into a $3 \mathrm{D}$ contour plot indicating the intensity minima (blue) at $10 \%$ and $25 \%$ of the maximum of the scale in the radial intensity slice. Note the axial extension is only one wavelength $532 \mathrm{~nm}$, i.e., exaggerated by a factor of $\approx 75$.

possible in crossed-beam dipole lattices where trap lengths are limited to the typical beam waists of $\approx 100 \mu \mathrm{m}$. For the helical scheme tunable axial confinement would be provided with harmonic magnetic fields.

The use of larger rings for trapping Bose-Einstein condensates has applications in rotational sensing [16], and more recently there have been rapid developments in small-scale optical traps for precise studies of superfluidity and the quantization of flow in rings $[\underline{17}, \underline{18}]$. The traps we show in Figs. 3 and $\underline{5}$ illustrate a way of extending the single ring traps of Refs. [17,18], allowing scalability to a large array of rings in which the flows in each ring could be stored as individual qubits for quantum processing.

A major advantage of the counterpropagating lattice geometry is that structures can be formed with subwavelength axial structure, and tight axial confinement can be achieved without additional fields-like the bright geometry discussed in Ref. [13]. The lattice is an interferometric superposition of counterpropagating beams, as
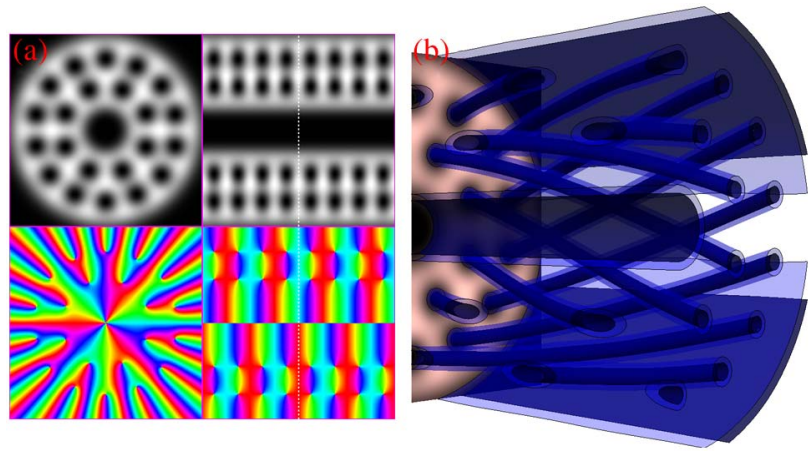

Fig. 2. (Color online) An "optical revolver" generated by a superposition of $100 \mathrm{~mW}$ of $\mathrm{LG}_{3}$ and $290 \mathrm{~mW}$ of $\mathrm{LG}_{25}$ with counterpropagating $190 \mathrm{~mW}$ of $\mathrm{LG}_{11}$, all other dimensions as for Fig. 1. Here, $R=0.26 \mathrm{~Hz}, U=60 \mu \mathrm{K}$. The inner helical dark core has a lead angle of $38 \mathrm{mrad}$ and leads to harmonic trap frequencies $183 \mathrm{kHz}$ and $6.9 \mathrm{kHz}$ in the axial and radial directions, respectively.
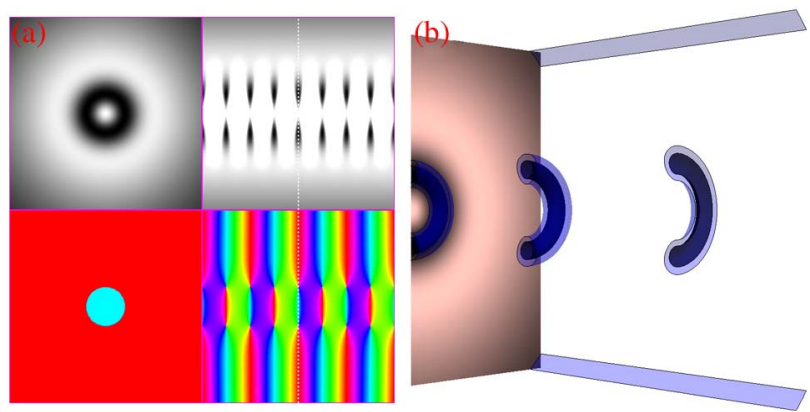

Fig. 3. (Color online) An axial lattice of rings formed by a $100 \mathrm{~mW} \mathrm{LG}_{0}$ beam (with the typical $5 \mu \mathrm{m}$ waist) counterpropagating with $1.0 \mathrm{~W}$ of $\mathrm{LG}_{0}$ beam (with a waist of $30 \mu \mathrm{m}$ ). Here, $R=0.18 \mathrm{~Hz}$ and $U=42 \mu \mathrm{K}$. Each dark ring has trap frequencies $370 \mathrm{kHz}$ and $4.9 \mathrm{kHz}$ in the axial and radial directions, respectively. This geometry is useful for storing multiple persistent currents or creating annular Tonks-Girardeau gases.

such although the shape of the lattice is not phasedependent, phase-stability is required to completely axially localize the optical traps. A trick used in optical lattices, to avoid aligning two separate beams with interferometric stability, is to retroreflect Gaussian beams with a mirror near the position of the trap. The node of the transverse electric field at the mirror ensures lattice stability if this mirror alone is stable.

Here we suggest (Fig. 6 ) the use of a phase grating near the trap location as this will have three advantages: the lattice axial location depends only on the grating stability, alignment of the trap relies only on successful angle alignment of the grating, and the grating itself can be used to change the LG mode superposition of the beam into the required counterpropagating mode to complete the lattice. In order to achieve sufficient resolution that the grating efficiently and accurately generates the required mode, the incoming beam must cover several hundred grating periods. This could be achieved in vacuo, in Littrow configuration, slightly away from the beam focus with a microfabricated grating substrate with a size of $\approx 200 \mu \mathrm{m}$ and grating period $\approx 1 \mu \mathrm{m}$ (ensuring a large diffraction angle so other diffracted orders do not affect the beam superposition). An ex vacuo grating would simplify alignment, and as long as an astigmatism-compensated
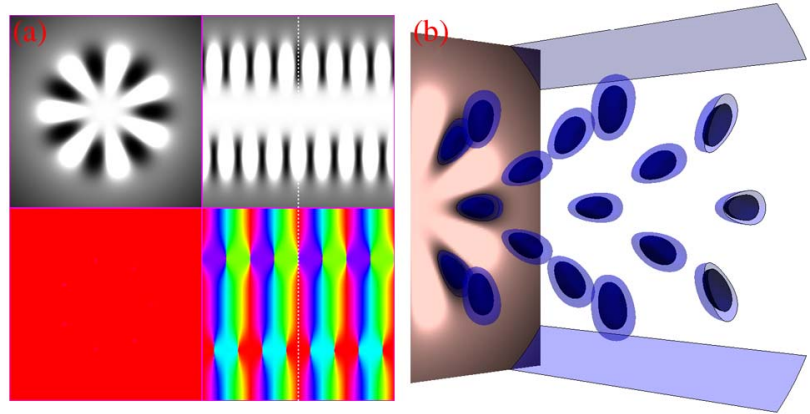

Fig. 4. (Color online) An axial lattice of ring lattices formed by a $100 \mathrm{~mW} \mathrm{LG}_{7}$ beam and $100 \mathrm{~mW} \mathrm{LG}_{-7}$ (with the typical $5 \mu \mathrm{m}$ waist) counterpropagating with $2.6 \mathrm{~W}$ of $\mathrm{LG}_{0}$ beam (with a waist of $30 \mu \mathrm{m}$ ). Here, $R=0.60 \mathrm{~Hz}$ and $U=140 \mu \mathrm{K}$. Each dark trap has an axial trap frequency of $550 \mathrm{kHz}$, and the trap is quartic rather than harmonic in the radial and azimuthal directionsuseful for exploring $2 \mathrm{D}$ quantum gases. 


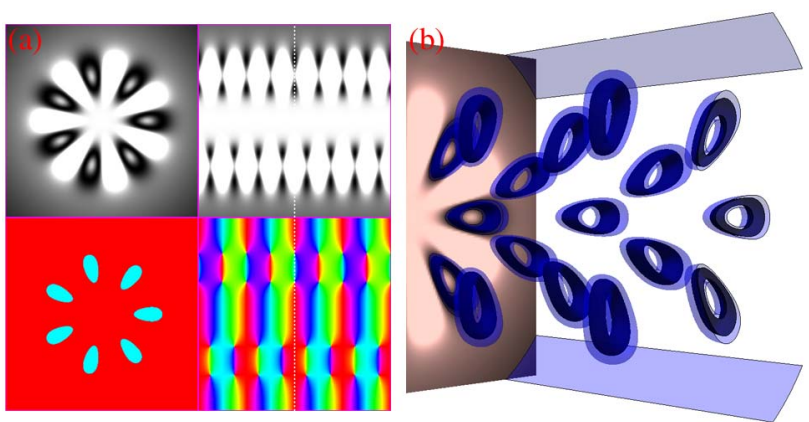

Fig. 5. (Color online) An axial lattice of ring lattices of rings, all parameters as in Fig. $\underline{4}$, except the counterpropagating Gaussian beam has power $650 \mathrm{~mW}$. Here, $R=0.15 \mathrm{~Hz}$ and $U=34 \mu \mathrm{K}$. Each dark ring has a spatially varying axial (radial) trap frequency of $260-290 \mathrm{kHz}(4.6-15 \mathrm{kHz})$.

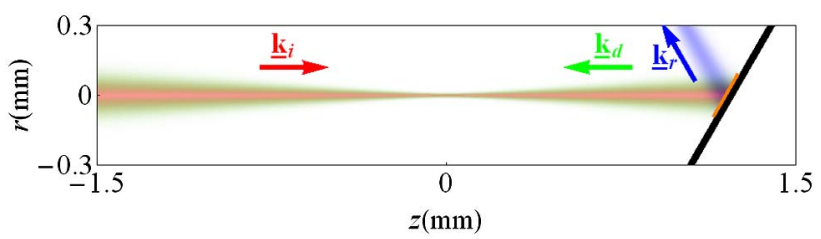

Fig. 6. (Color online) An incident (red, $\underline{\mathbf{k}}_{i}$ ) laser beam diffracts from a $2 \mathrm{D}$ phase grating (with a lens) into counterpropagating (green, $\underline{\mathbf{k}}_{d}$ ) and discarded reflected (blue, $\underline{\mathbf{k}}_{r}$ ) orders.

zoom lens is used [19], a spatial light modulator could be used as a means to simply program different phase gratings and change lattice geometries.

The scheme's robustness to experimental imperfections is vital. Laser pointing stability is paramount, precluding ion lasers in favor of fiber-coupled tapered amplifiers or doubled DPSS/fiber lasers. Counterpropagating beam misalignment can be minimized using a small aperture near the fiber output, or by fiber-recoupling counterpropagating light. We estimate this misalignment, via ray-tracing a simple beam line, to be angular (and associated radial) values of $\approx 1 \mathrm{mrad}$ and $1 \mu \mathrm{m}$, at the trap location. This leads to noticeable changes in the total intensity pattern, particularly for Figs. 1(a) and 2(a) due to the small counterpropagating waists. However, the dark trapping potentials in the 3D contour plots [Figs. 1-5(b)] remain largely unchanged, even with relative mode power changes of $\approx 20 \%$. In conclusion, we have illustrated several new dark counterpropagating optical trapping geometries and presented a realistic, robust scheme for their experimental generation. The dark nature of these traps mean that their zero differential light shifts can be used in atom interferometry, and they also have applications in extending Tonks-Girardeau gases, parallelization of superflow and quantum information storage.

The author is grateful for stimulating discussions with E. Riis, P. F. Griffin, S. Franke-Arnold, and G. Walker.

\section{References}

1. M. R. Andrews, C. G. Townsend, H.-J. Miesner, D. S. Durfee, D. M. Kurn, and W. Ketterle, Science 275, 637 (1997).

2. M. E. Zawadzki, P. F. Griffin, E. Riis, and A. S. Arnold, Phys. Rev. A 81, 043608 (2010).

3. K. W. Madison, F. Chevy, W. Wohlleben, and J. Dalibard, Phys. Rev. Lett. 84, 806 (2000).

4. I. Bloch, Nat. Phys. 1, 23 (2005).

5. K. Bongs, S. Burger, S. Dettmer, D. Hellweg, J. Arlt, W. Ertmer, and K. Sengstock, Phys. Rev. A 63, 031602(R) (2001).

6. S. Franke-Arnold and A. S. Arnold, Am. Sci. 96, 226 (2008).

7. R. Ozeri, L. Khaykovich, and N. Davidson, Phys. Rev. A 59, R1750 (1999).

8. J. Arlt and M. J. Padgett, Opt. Lett. 25, 191 (2000).

9. S. E. Olson, M. L. Terraciano, M. Bashkansky, and F. K. Fatemi, Phys. Rev. A 76, 061404(R) (2007).

10. S. Franke-Arnold, J. Leach, M. J. Padgett, V. E. Lembessis, D. Ellinas, A. J. Wright, J. M. Girkin, P. Öhberg, and A. S. Arnold, Opt. Express 15, 8619 (2007).

11. X. He, P. Xu, J. Wang, and M. Zhan, Opt. Express 17, 21007 (2009).

12. E. Courtade, O. Houde, J.-F. Clément, P. Verkerk, and D. Hennnequin, Phys. Rev. A 74, 031403 (2006).

13. L. Amico, A. Osterloh, and F. Cataliotti, Phys. Rev. Lett. 95, 063201 (2005).

14. M. Bhattacharya, Opt. Commun. 279, 219 (2007).

15. S. Palzer, C. Zipkes, C. Sias, and M. Köhl, Phys. Rev. Lett. 103, 150601 (2009), and references therein.

16. P. F. Griffin, E. Riis, and A. S. Arnold, Phys. Rev. A 77, 051402(R) (2008), and references therein.

17. A. Ramanathan, K. C. Wright, S. R. Muniz, M. Zelan, W. T. Hill, III, C. J. Lobb, K. Helmerson, W. D. Phillips, and G. K. Campbell, Phys. Rev. Lett. 106, 130401 (2011).

18. S. Moulder, S. Beattie, R. P. Smith, N. Tammuz, and Z. Hadzibabic, arXiv: 1112.0334.

19. J. F. Sherson, C. Weitenberg, M. Endres, M. Cheneau, I. Bloch, and S. Kuhr, Nature 467, 68 (2010). 